\title{
Beyond complex Langevin equations: from simple examples to positive representation of Feynman path integrals directly in the Minkowski time
}

\author{
Jacek Wosiek \\ M. Smoluchowski Institute of Physics, Jagellonian University, \\ Eojasiewicza St. 11, 30-348 Krakow, Poland \\ E-mail: wosiek@th.if.uj.edu.pl
}

ABSTRACT: A positive representation for an arbitrary complex, gaussian weight is derived and used to construct a statistical formulation of gaussian path integrals directly in the Minkowski time. The positivity of Minkowski weights is achieved by doubling the number of real variables. The continuum limit of the new representation exists only if some of the additional couplings tend to infinity and are tuned in a specific way. The construction is then successfully applied to three quantum mechanical examples including a particle in a constant magnetic field - a simplest prototype of a Wilson line. Further generalizations are shortly discussed and an intriguing interpretation of new variables is alluded to.

Keywords: Field Theories in Lower Dimensions, Lattice Quantum Field Theory, Stochastic Processes, Wilson, 't Hooft and Polyakov loops

ARXIV EPRINT: 1511.09114 


\section{Contents}

1 Introduction and the main idea $\quad 1$

2 Single integral - gaussian case $\quad 3$

3 Single integral - nonlinear case 4

4 Many variables $\quad 6$

5 The continuum limit $\quad 7$

$\begin{array}{lll}5.1 \text { A free particle } & 7\end{array}$

5.2 A harmonic oscillator 8

6 Applications $\quad 10$

$\begin{array}{lll}6.1 \text { A free particle } & 10\end{array}$

6.2 A harmonic oscillator $\quad 11$

$\begin{array}{lll}\text { 6.3 Charged particle in a constant magnetic field } & 13\end{array}$

$\begin{array}{lll}7 & \text { Summary and conclusions } & 14\end{array}$

\section{Introduction and the main idea}

Stochastic quantization [1, 2] based on complex Langevin equations [3, 4] has attracted again a new wave of interest. This was caused by reported lately progress in simulating lattice QCD at finite chemical potential [5, 6]. At the same time the old issues [7-9], the approach suffered with, resurfaced again $[10,11]$ only to emphasize the difficulty with theoretical foundations of the method. The latters are much more satisfactory nowadays $[12,13]$, revealing quite complicated nature of the problem.

In this article a positive representation [14], equivalent to the complex gaussian distribution in the complex Langevin approach, is studied in detail. The problem is not new and its classic, by now, solution is known for a long time [7]. The novelty of the present result is that it provides, an independent of any stochastic process, positive representation for an arbitrary, complex value of the inverse dispersion parameter $\sigma$, while the original one applies for $\mathcal{R} e \sigma>0$ only. In particular the new solution works also for purely imaginary $\sigma .{ }^{1}$ This opens a possibility of a positive representation for Feynman path integrals directly in the Minkowski time - the quest which still awaits its resolution.

\footnotetext{
${ }^{1}$ Somewhat different solution of the general complex gaussian model exists in the literature [15]. It is entangled with the particular modification (kernel method) of the stochastic process for a general complex $\sigma$. Consequently the analytic solution quoted there depends on the kernel employed. Present solution does not refer to any stochastic process, hence it is independent of any additional parameters.
} 
Indeed, by the direct extension the one degree of freedom trick, it is shown that such a description is possible. It is constructed and applied to few quantum mechanical textbook cases. Noteworthy, the construction covers also the path integral description of a particle in a constant magnetic field - a problem which does not have a positive representation after the Wick rotation.

In 2002 Weingarten [16] has addressed analogous question in more general terms and has proved that the positive densities actually exist for a wide class of complex probabilities. Nevertheless no practical construction of such distributions was attempted even for the gaussian case (see however $[17,18]$ ). Moreover, the continuum limit was explicitly not discussed. As will be seen below, existence of the continuum limit plays an essential role in the present solution.

To begin with, we illustrate the main trick for a single integral. In general, quantum averages result from weighting observables with complex functions $\rho(x) \equiv e^{-S(x)}$, rather than with positive probabilities. The complex Langevin approach can in principle address this difficulty by replacing a complex average with the statistical average over the complex stochastic process determined by a complex action $S(x)$

$$
\frac{\int f(x) e^{-S(x)} d x}{\int e^{-S(x)} d x}=\frac{\iint f(x+i y) P(x, y) d x d y}{\iint P(x, y) d x d y},
$$

with $P(x, y)$ being the distribution of the above process at large Langevin time. While this idea works well and has been proven for real actions, it still rises some theoretical questions and encounters practical difficulties in the complex case, even though a much more complete theoretical understanding of the problem has been achieved lately $[12,13]$.

Instead we have constructed $P(x, y)$ directly using (1.1) as a starting point and avoiding any reference to stochastic processes and associated Fokker-Planck equations. The derivation works as follows. Introduce two independent, complex variables

$$
z=x+i y, \quad \bar{z}=x-i y
$$

and rewrite (1.1) as

$$
\begin{gathered}
\frac{\int_{R} f(x) \rho(x) d x}{\int_{R} \rho(x) d x}=\frac{\int_{\Gamma_{z}} f(z) \rho(z) d z}{\int_{\Gamma_{z}} \rho(z) d z}= \\
\frac{\int_{\Gamma_{z}} \int_{\Gamma_{\bar{z}}} f(z) P(z, \bar{z}) d z d \bar{z}}{\int_{\Gamma_{z}} \int_{\Gamma_{\bar{z}}} P(z, \bar{z}) d z d \bar{z}}=\frac{\int_{R^{2}} f(x+i y) P(x, y) d x d y}{\int_{R^{2}} P(x, y) d x d y} .
\end{gathered}
$$

Contours $\Gamma_{z}$ and $\Gamma_{\bar{z}}$ are such that the integrals exists. Above equations will be satisfied provided we find $P(z, \bar{z})$ such that

$$
\rho(z)=\int_{\Gamma_{\bar{z}}} P(z, \bar{z}) d \bar{z} .
$$

This is the key relation of the new approach. On one side it provides a simple connection between a complex weight $\rho$ and $P$, while on the other leaves us a freedom to satisfy positivity and normalizability of $P(z, \bar{z})$ restricted to $R^{2}$ by (1.2). 
The paper is organized as follows: in the next section we apply above construction to the gaussian case thereby generalizing solution of ref. [7] to an arbitrary complex inverse dispersion parameter $\sigma$. Section 4 contains a first nontrivial application to the nonlinear action showing that the approach is not necessarily restricted to the linear systems. In section 5 we extend the gaussian case with purely imaginary $\sigma$ to arbitrary number of variables, apply it to the Minkowski path integrals and derive conditions for the existence of the continuum limit. Section 6 contains concrete applications to three quantum mechanical problems thereby constructing for the first time positive representations for Minkowski path integrals. Finally we end with summary and the outlook in section 7 .

\section{Single integral - gaussian case}

For the gaussian action we take

$$
P(z, \bar{z})=\frac{i}{2} \exp \left(-\left(a^{*} z^{2}+2 b z \bar{z}+a \bar{z}^{2}\right)\right), \quad a=\alpha+i \beta, b=b^{*},
$$

or in terms of $x$ and $y$,

$$
P(x, y)=\exp \left(-2\left((b+\alpha) x^{2}+2 \beta x y+(b-\alpha) y^{2}\right)\right)
$$

which is positive and normalizable for $|a|<b$. Corresponding complex density follows from $(1.4)^{2}$

$$
\rho(z)=\int_{\Gamma_{\bar{z}}} P(z, \bar{z}) d \bar{z}=\frac{1}{2} \sqrt{\frac{\pi}{-a}} \exp \left(-\sigma z^{2}\right), \quad \sigma=\frac{|a|^{2}-b^{2}}{a},
$$

and indeed is given by a gaussian with arbitrary complex $\sigma$.

It is a simple exercise to confirm eq. (1.3) for power-like observables, e.g. by calculating the generating function in both representations.

For $\operatorname{Re} \sigma>0$ the contour $\Gamma_{z}$ can be rotated into the real axis and eq. (1.1) established. However (2.2) is more general than the original solution [7] since it provides the positive representation for arbitrary complex $a$, or equivalently $\sigma \in C$. For some $\sigma$, for example $\sigma \in R, \sigma<0$, the contour $\Gamma_{z}$ cannot be rotated back into the real axis. Then eq. (2.2) gives the positive and normalizable representation for the averages along the allowed $\Gamma_{z}$, or in another words, for the analytic continuation of the divergent, along the real axis, expressions.

To conclude this section we discuss two interesting special cases.

For real and negative $\sigma$, the complex density blows up along the real axis. On the other hand the distribution $P(x, y)$ is positive and normalizable at $\alpha>0$ and $\beta=0$ producing the correct average over the "divergent" distribution $\rho$. This explains a "striking example" observed in the literature [19], namely that, upon change of variables, the complex Langevin simulation based on old solution of ref. [7] actually has the correct fixed point also for negative $\mathcal{R} e \sigma$ (where the distribution is non-normalizable). The answer is that their

\footnotetext{
${ }^{2}$ For $\Gamma_{\bar{z}}$ one can choose a straight line contained in a wedge determined by a phase of $a$.
} 
positive distribution used until now is part of a richer structure (2.2), which accommodates negative $\mathcal{R} e \sigma$ as well.

Similarly, the complex density $\rho(z)$ for purely imaginary $\sigma$ is readily represented by the positive distribution $P(x, y)$, which is perfectly well defined at $\alpha=0$ and arbitrary $\beta$, as long as $|\beta|<b$. This opens an exciting possibility of positive representations for Feynman path integrals directly in the Minkowski time, which is explored in detail in this paper.

In both cases the original density [7] does not exists.

\section{Single integral - nonlinear case}

Another possible solution of (1.1) obtains if we start from the action

$$
S_{4}(z, \bar{z})=\left(a^{*} z^{2}+2 b z \bar{z}+a \bar{z}^{2}\right)\left(c^{*} z^{2}+2 d z \bar{z}+c \bar{z}^{2}\right),
$$

with complex $a$ and $c$ and real $b \gtrless|a|$ and $d \gtrless|c|$. The density $P(x, y)$ is again positive and normalizable on the $x, y$ plane. To derive $\rho(z)$ introduce an arbitrary shift parameter $e$ and change the variables. This gives

$$
S_{4}(z, \bar{z}=u-e z)=A_{0} z^{4}+A_{1} z^{3} u+A_{2} z^{2} u^{2}+A_{3} z u^{3}+A_{4} u^{4},
$$

with

$$
\begin{aligned}
& A_{4}=a c, \\
& A_{3}=2(a d+b c)-4 a c e, \\
& A_{2}=a^{*} c+c^{*} a+4 b d-6 e(a d+b c)+6 a c e^{2}, \\
& A_{1}=2\left(b c^{*}+a^{*} d\right)-2 e\left(a^{*} c+a c^{*}+4 b d\right)+6 e^{2}(b c+a d)-4 e^{3} a c, \\
& A_{0}=\left(a^{*}-2 b e+a e^{2}\right)\left(c^{*}-2 d e+c e^{2}\right) .
\end{aligned}
$$

Now choose $e$ such that $A_{3}=0$. The coefficients become

$$
\begin{aligned}
& A_{4}=a c, \\
& A_{2}=\frac{1}{2 a c}\left(2 a^{2}\left(|c|^{2}-d^{2}\right)+2 c^{2}\left(|a|^{2}-b^{2}\right)-(a d-b c)^{2}\right), \\
& A_{1}=\frac{1}{a^{2} c^{2}}(a d-b c)\left(a^{2}\left(|c|^{2}-d^{2}\right)-c^{2}\left(|a|^{2}-b^{2}\right)\right), \\
& A_{0}=\frac{1}{16 a^{3} c^{3}}\left(4 c^{2}\left(|a|^{2}-b^{2}\right)+(a d-b c)^{2}\right)\left(4 a^{2}\left(|c|^{2}-d^{2}\right)+(a d-b c)^{2}\right) .
\end{aligned}
$$

Then $A_{1}$ can be also eliminated setting

$$
c=\frac{d}{b} a,
$$

which essentially reduces $S_{4}(z, \bar{z})$ to a square. Remaining coefficients simplify

$$
\begin{aligned}
& A_{4}=\frac{d}{b} a^{2}, \\
& A_{2}=2 \frac{d}{b}\left(|a|^{2}-b^{2}\right), \\
& A_{0}=\frac{d}{b}\left(|a|^{2}-b^{2}\right)^{2} \frac{1}{a^{2}} .
\end{aligned}
$$


The complex density $\rho_{4}(z)$ can be then obtained in a closed form as

$$
\begin{aligned}
\rho_{4}(z) & =\frac{i}{2} \int_{\Gamma_{\bar{z}}} d \bar{z} e^{-S_{4}(z, \bar{z})} \\
& =\frac{i}{2} \exp \left(-A_{0} z^{4}\right) \int_{\Gamma_{u}} d u \exp \left(-A_{4} u^{4}-A_{2} z^{2} u^{2}\right) \\
& =\frac{i}{2}\left(\frac{b}{2 d a^{2}}\right)^{\frac{1}{4}} \exp \left(-\sigma z^{4}\right)\left(\sigma z^{4}\right)^{\frac{1}{4}} K_{\frac{1}{4}}\left(\sigma z^{4}\right),
\end{aligned}
$$

with an arbitrary complex

$$
\sigma=\frac{d\left(b^{2}-|a|^{2}\right)^{2}}{2 b a^{2}}
$$

As before all contours (here and below) are such that the integrals exists. Again one can choose straight lines with slopes determined by the phase of $a$.

It is a simple exercise to show that normalization of both densities is the same:

$$
\begin{aligned}
\int_{\Gamma_{z}} \rho_{4}(z) d z & =\frac{i}{2}\left(\frac{b}{2 d a^{2}}\right)^{\frac{1}{4}} \int_{\Gamma_{z}} \exp \left(-\sigma z^{4}\right)\left(\sigma z^{4}\right)^{\frac{1}{4}} K_{\frac{1}{4}}\left(\sigma z^{4}\right)= \\
\frac{\pi^{\frac{3}{2}}}{4} \sqrt{\frac{b}{d\left(b^{2}-|a|^{2}\right)}} & =\int_{R^{2}} d x d y e^{-4 \frac{d}{b}\left((b+\alpha) x^{2}+2 \beta x y+(b-\alpha) y^{2}\right)^{2}}=\int_{R^{2}} d x d y P_{4}(x, y) .
\end{aligned}
$$

The difference however, being that while on the l.h.s. the density $\rho$ is in general complex, and contour $\Gamma_{z}$ has to be adjusted depending on a phase of $\sigma$, the integral on the r.h.s. is always over $R^{2}$ and the density $P_{4}(x, y)$ is positive and normalizable for all complex $\sigma$.

The same applies to higher moments:

$$
\int_{\Gamma_{z}} z^{n} \rho_{4}(z) d z=\int_{R^{2}} d x d y(x+i y)^{n} P_{4}(x, y) .
$$

In fact the construction works for a larger range of parameters that in the gaussian case since the condition $|a|<b$ can be released.

The density (3.1) has the simple leading asymptotics

$$
\rho_{4}(z) \sim e^{\left(-2 \sigma z^{4}\right)}, \quad z \longrightarrow \infty
$$

and therefore might be of some practical interest (e.g. in optimizing some reweighting algorithms). The main point of this example is however, that the original idea, namely constructing positive representations with the aid of a second variable, applies not only to the gaussian cases, therefore it may indicate the existence of some unexplored yet structures.

Obviously there is a lot of freedom in choosing an initial action. It remains to be seen to what extent this freedom allows to derive complex densities of wider physical interest. In present approach, the freedom results from the nonuniqueness in inverting the relation (1.4). It would be interesting to study if it is connected with the one present in other approaches based directly on stochastic processes [19] 


\section{Many variables}

For the action we take $N$ copies of (2.1) and add the nearest neighbour couplings, with periodic boundary conditions in $z_{i}$ and $\bar{z}_{i}: z_{N+1}=z_{1}, \bar{z}_{N+1}=\bar{z}_{1}, z_{0}=z_{N}, \bar{z}_{0}=\bar{z}_{N}$, $a, c \in C, b \in R$,

$$
S_{N}(z, \bar{z})=\sum_{i=1}^{N} a \bar{z}_{i}^{2}+2 b \bar{z}_{i} z_{i}+2 c \bar{z}_{i} z_{i+1}+2 c^{*} z_{i} \bar{z}_{i+1}+a^{*} z_{i}^{2}
$$

The complex density $\rho(z)$ results from integrating $P_{N}(z, \bar{z})$ over all $\bar{z}$ variables

$$
\rho(z)=\int \prod_{i=1}^{N} d \bar{z}_{i} P(z, \bar{z})=\left(\frac{i}{2}\right)^{N} \int \prod_{i=1}^{N} d \bar{z}_{i} \exp \left(-S_{N}(z, \bar{z})\right) .
$$

The integration is elementary and one obtains for the effective action

$$
\begin{aligned}
S_{N}^{\rho} \equiv & -\log \left\{\left(\frac{-4 a}{\pi}\right)^{\frac{N}{2}} \rho(\{z\})\right\}=\sum_{i=1}^{N} \frac{B}{2 a}\left(z_{i}^{2}+2 \frac{2 b\left(c+c^{*}\right)}{B} z_{i} z_{i+1}+z_{i+1}^{2}\right) \\
& +\frac{2 c c^{*}}{a}\left(z_{i-1} z_{i+1}-z_{i}^{2}\right), \quad B=b^{2}+\left(c+c^{*}\right)^{2}-|a|^{2} .
\end{aligned}
$$

If we set $c$ to be real and require

$$
2 c=2 \gamma=-b+|a|,
$$

the effective action simplifies to

$$
-S_{N}^{\rho}(z)=\mathcal{A} \sum_{i=1}^{N}\left(z_{i}-z_{i+1}\right)^{2}-r\left(z_{i-1}-z_{i+1}\right)^{2}, \quad \mathcal{A}=\frac{b(b-|a|)}{a}, \quad r=\frac{b-|a|}{4 b} .
$$

This is reminiscent of the discretized Feynman action for a free particle. The second term however, even though similar to the first one, requires further attention and will be discussed shortly.

Leaving this for a moment let us check now the positivity and normalizability of the corresponding probability density $P_{N}(x, y)$ on $R^{2 N}$. In terms of real and imaginary parts of $z_{i}$ the action (4.1) reads

$$
S_{N}(x, y)=2 \sum_{i=1}^{N}(b+\alpha) x_{i}^{2}+2 \beta x_{i} y_{i}+(b-\alpha) y_{i}^{2}+2 \gamma\left(x_{i} x_{i+1}+y_{i} y_{i+1}\right) .
$$

Hence

$$
P_{N}(x, y)=\exp \left(-S_{N}(x, y)\right)
$$

is obviously positive. With $\gamma$ given by (4.3), all $2 N$ eigenvalues are non-negative - there are no divergent directions. There is one zero mode associated with the translational invariance, however this is usual and can be dealt with by standard means. 


\section{The continuum limit}

\subsection{A free particle}

The action (4.4) does not agree with the standard, discretized action of a free particle

$$
S_{N}^{\mathrm{free}}=\frac{i m}{2 \hbar \epsilon} \sum_{i=1}^{N}\left(z_{i+1}-z_{i}\right)^{2},
$$

except at $r=0$. To see better the effect of the next-to-nearest $(n n)$ term, we analyze in detail the large $N$ behaviour of, e.g., the propagator

$$
K_{N}\left(z_{N}, z_{1}\right)=e^{-\mathcal{A}\left(z_{N}-z_{1}\right)^{2}} I_{N}\left(z_{N}, z_{1}\right)=e^{-\mathcal{A}\left(z_{N}-z_{1}\right)^{2}} \int d z_{2} \ldots d z_{N-1} e^{-S_{N}^{\rho}\left(z_{1}, \ldots, z_{N}\right)} .
$$

For simplicity we shall work in the "exponential accuracy", i.e. ignore all prefactors. They can be dealt with by usual methods and do not affect any conclusions drawn here. We also rescale temporarily all variables $\mathcal{A} z_{i}^{2} \rightarrow z_{i}^{2}$ to further simplify all expressions. The integral (5.2) can be calculated recursively, $k=2,3, \ldots, N-1$,

$$
I_{k}^{(N)}\left(z_{N}, z_{1} ; v, w\right)=\int d u I_{k-1}^{(N)}\left(z_{N}, z_{1} ; u, v\right) e^{(u-v)^{2}-r(u-w)^{2}},
$$

with the initial condition

$$
I_{1}^{(N)}\left(z_{N}, z_{1}, u, v\right)=\exp \left(\left(z_{1}-u\right)^{2}-r\left(z_{1}-v\right)^{2}-r\left(z_{N}-u\right)^{2}\right) .
$$

The propagator obtains after $N-2$ steps

$$
I_{N}\left(z_{N}, z_{1}\right)=\left.I_{N-1}^{(N)}\left(z_{N}, z_{1} ; u, v\right)\right|_{(u, v)->\left(z_{N}, z_{1}\right)} .
$$

It is straightforward to derive recursion relations for the exponents of $I_{k}^{(N)}$. Define

$$
W_{k}(u, v)=\log I_{k}^{(N)}\left(z_{N}, z_{1} ; u, v\right)=a_{k} u^{2}+2 b_{k} u v+c_{k} v^{2}+2 d_{k} u+2 e_{k} v+f_{k},
$$

then

$$
\begin{aligned}
a_{k+1} & =1+c_{k}+\frac{2 b_{k}-b_{k}^{2}-1}{1-r+a_{k}}, & b_{k+1} & =\frac{r-r b_{k}}{1-r+a_{k}}, \\
c_{k+1} & =1-\frac{1}{1-r+a_{k}}, & d_{k+1} & =e_{k}+\frac{d_{k}-b_{k} d_{k}}{1-r+a_{k}}, \\
e_{k+1} & =\frac{-r d_{k}}{1-r+a_{k}}, & f_{k+1} & =f_{k}-\frac{d_{k}^{2}}{1-r+a_{k}},
\end{aligned}
$$

with the initial conditions implied by (5.3)

$$
\begin{aligned}
& a_{1}=1-r, \quad b_{1}=0, \quad c_{1}=-r, \\
& d_{1}=-z_{1}+r z_{N}, \quad e_{1}=r z_{1}, \quad f_{1}=z_{1}^{2}-r z_{1}^{2}-r z_{N}^{2} .
\end{aligned}
$$


Results are the following: $W_{N}\left(z_{N}, z_{1}\right)$ is quadratic and depends only on the difference

$$
W_{N}\left(z_{N}, z_{1}\right)=\sigma_{N}(r)\left(z_{N}-z_{1}\right)^{2}
$$

as required by the translational invariance. The coefficient $\sigma_{N}$ is the ratio of two polynomials

$$
\sigma_{N}(r)=\frac{P_{N}(r)}{Q_{N}(r)}
$$

and can be expanded for large $N$ as

$$
\sigma_{N}(r)=v_{0}(r)+\frac{v_{1}(r)}{N}+\frac{v_{2}(r)}{N^{2}}+\ldots
$$

At $r=0$, all coefficients $v_{i}$ vanish except of $v_{1}(0)=1$. This is the standard Feynman case without the $n n$ term, cf. (4.4), (5.1). For $r \neq 0$ however all $v_{i}$ do not vanish, in particular $v_{0} \neq 0$. This precludes existence of the continuum limit

$$
N \rightarrow \infty, \quad N \epsilon \text { fixed }
$$

which requires

$$
\mathcal{A} \sigma_{N}(r) \rightarrow \text { const. } \quad \mathcal{A} \sim \frac{1}{\epsilon}
$$

as follows from (5.1). In principle one might consider renormalizing the divergent term away - the possibility which should be looked at in more detail. However we choose here a simpler solution. Both constraints, namely

$$
\mathcal{A}=\frac{b(b-|a|)}{a} \rightarrow \frac{i m}{2 \hbar \epsilon}, \text { and } r=\frac{b-|a|}{4 b} \rightarrow 0,
$$

can be satisfied in the limit (referred from now on as $\lim _{1}$ )

$$
|a|, b \rightarrow \infty, b-|a|=\frac{m}{2 \hbar \epsilon}=\text { const. } \equiv d, \quad a=-i|a| .
$$

This completes the construction of the positive representation for the path integral of a free particle directly in the Minkowski time.

All quantum averages can now be obtained by weighting suitable, i.e. complex in general, observables with the positive and normalizable distribution (4.6), and then taking the limit (5.6) followed by the continuum limit (5.5). Subsequent applications illustrate how this works in practice.

\subsection{A harmonic oscillator}

Interestingly this case is also covered by the action (4.1), (4.5). The only difference lies in the scaling laws imposed during the first limiting transition (5.6). To see this consider the first term in eq. (4.2), for real $c=\gamma$,

$$
D z_{i}^{2}+2 E z_{i} z_{i+1}+D z_{i+1}^{2}, \quad D=\frac{b^{2}+4 \gamma^{2}-|a|^{2}}{2 a}, \quad E=\frac{2 b \gamma}{a} .
$$


Rewrite it as

$$
-E\left(\left(z_{i+1}-z_{i}\right)^{2}-\left(\frac{D}{E}+1\right)\left(z_{i}^{2}+z_{i+1}^{2}\right)\right),
$$

and compare with an analogous term in the discretization of the Minkowski action of a harmonic oscillator

$$
\frac{i m}{2 \hbar \epsilon}\left(\left(x_{1}-x_{2}\right)^{2}-\frac{\omega^{2} \epsilon^{2}}{2}\left(x_{1}^{2}+x_{2}^{2}\right)\right) .
$$

Therefore, the general positive distribution (4.5) in $2 N$ real variables describes a harmonic oscillator if we identify

$$
-\frac{2 b \gamma}{a}=\frac{i m}{2 \hbar \epsilon}, \quad \frac{b^{2}+4 \gamma^{2}-|a|^{2}}{4 b \gamma}+1=\frac{\omega^{2} \epsilon^{2}}{2} .
$$

Similarly to the free particle case, the $n n$ terms will vanish for large $|a|$ and $b$. However the limit has to be taken along the trajectory (5.7). A possible parametrization in terms of one independent variable $\nu$, is

$$
a=-i|a|, \quad b=\frac{\mu}{\nu}, \quad|a|=\frac{\mu}{\nu} \zeta(\nu, \rho), \quad 2 \gamma=-\mu \zeta(\nu, \rho),
$$

where

$$
\zeta(\nu, \rho)=\frac{\sqrt{1-2 \nu^{2} \rho+\nu^{2} \rho^{2}}-\nu(1-\rho)}{1-\nu^{2}},
$$

and $\mu$ and $\rho$ depend on $N$ and parameters of the harmonic oscillator in the continuum

$$
\rho=\frac{\omega^{2} T^{2}}{2(N-1)^{2}}, \quad \mu=\frac{m(N-1)}{2 \hbar T} .
$$

Vanishing of the $n n$ term is achieved by taking $\nu \rightarrow 0$.

This is the main modification compared to the free particle case. With the first limit taken along the trajectory (5.8) the action (4.5) provides a positive representation for Minkowski path integral of a one-dimensional harmonic oscillator.

However now one eigenvalue of (4.5) becomes "weakly negative" and the procedure requires additional care. This is the familiar zero eigenvalue encountered before, which for general $\gamma$ and imaginary $a$ reads

$$
\lambda_{0}=2(b-|a|+2 \gamma)
$$

with the corresponding eigenvector having all equal components. In the free particle case (4.3) $\lambda_{0}=0$ reflecting the translational symmetry. Along the new trajectory (5.8) however, $\lambda_{0}$ does not vanish and is negative. Moreover, after the first limit

$$
\lim _{\nu \rightarrow 0} \lambda_{0}=-\frac{m \omega^{2} T}{4 \hbar(N-1)}
$$

and tends to zero with $N \rightarrow \infty$. The eigenvector remains the same for arbitrary $\gamma$ and becomes the true zero mode in the continuum limit. That is why the mode was called "weakly negative". Therefore one can treat it similarly to the usual zero modes, e.g. fix it. In fact, a negative mode is simpler than the zero mode since moments of divergent distributions can be defined by the analytic continuation which provides a regularization of the divergent integral. Both ways do not affect the continuum limit as will be seen in the following applications. 


\begin{tabular}{|ccc|}
\hline$N$ & $\sigma_{N}(-i(b-d), b)$ & $\lim _{1}$ \\
\hline 5 & $\frac{i d\left(16 b^{2}+28 b d-19 d^{2}\right)}{8(8 b-3 d)(b-d)}$ & $i \frac{d}{4}$ \\
8 & $\frac{i d\left(16 b^{4}+40 b^{3} d-70 b^{2} d^{2}+23 b d^{3}-d^{4}\right)}{(b-d)\left(112 b^{3}-120 b^{2} d+30 b d^{2}-d^{3}\right)}$ & $i \frac{d}{7}$ \\
11 & $\frac{i d\left(1024 b^{5}+3328 b^{4}-9472 b^{3} d^{2}+6832 b^{2} d^{3}-1700 b d^{4}+109 d^{5}\right)}{(b-d)\left(1280 b^{4}-2304 b^{3} d+134 b^{2} d^{2}-280 b d^{3}+15 d^{4}\right)}$ & $i \frac{d}{10}$ \\
\hline
\end{tabular}

Table 1. The slope of the free propagator (6.2) and its limiting value for few discretizations.

\section{Applications}

\subsection{A free particle}

First, we shall calculate the free propagator integrating explicitly the new representation (4.5). The discretized kernel (5.2) reads

$$
K_{N}\left(z_{N}, z_{1}\right)=e^{-\mathcal{A}\left(z_{1}-z_{N}\right)^{2}} \int d \bar{z}_{1} \prod_{j=2}^{N-1} d x_{j} d y_{j} d \bar{z}_{N} \exp \left(-X^{T} M X\right) .
$$

The first factor takes away an additional contribution hidden in $S_{N}(4.5)$ due to the periodic boundary conditions as explicitly seen in (4.4). Since $z_{1}$ and $z_{N}$ are fixed, the first and the last integrals have to be done over $\bar{z}_{1}$ and $\bar{z}_{N}$ and not over the real coordinates. This is part of the construction: only complete traces are represented by integrals of positive distributions over the real variables, while deriving quantum amplitudes at fixed end-point requires integration over the corresponding complex, barred variables. Consequently $X$ is the vector of all variables, $X^{T}=\left(z_{1}, \bar{z}_{1}, x_{2}, y_{2}, x_{3} \ldots, y_{N-1}, z_{N}, \bar{z}_{N}\right)$, and $M$ is the matrix of (4.5) in this mixed representation. Gaussian integration is simple and one obtains up to a prefactor

$$
K_{N}\left(z_{N}, z_{1}\right) \sim \exp \left(\sigma_{N}(a, b)\left(z_{N}-z_{1}\right)^{2}\right),
$$

with $\sigma_{N}(a, b)$ given in table 1 for few values of $N$, and

$$
a=-i|a|, \quad|a|=b-d, d=\frac{m}{2 \hbar \epsilon} .
$$

Results after the first limit (5.6) are given in the third column. Indeed, as discussed in section 3 , the $v_{0}$ term (cf. (6.2)) does not survive and the limiting $\sigma_{N}$ has the appropriate large $N$ behaviour

$$
\lim _{b \rightarrow \infty} \sigma_{N}(-i(b-d), b)=\frac{i d}{N-1},
$$

which assures the correct and well known form for the Feynman kernel.

$$
\lim _{N \rightarrow \infty} K_{N} \sim \exp \left(\frac{i m}{2 \hbar} \frac{\left(z_{N}-z_{1}\right)^{2}}{T}\right) .
$$

This can be analytically continued to the real axes.

As a second example we calculate the average $\left\langle x^{2}(t)\right\rangle$ with the new representation. Physically this is the dispersion of a Minkowski path of a free particle at time $t$. The 
particle is constrained to start from, and return to, the origin after time $\mathrm{T}$. The continuum result,

$$
\left\langle x^{2}(t)\right\rangle=\frac{\int d x K(0, x ; T-t) x^{2} K(x, 0 ; t)}{K(0,0 ; T)}=\frac{i \hbar}{m} \frac{t(T-t)}{T},
$$

is purely imaginary and shows the famous statistical broadening of quantum paths as we move away from the fixed initial/final end points.

In our case this is again covered by (6.1), with $M$ replaced by its reduction $R$ which does not involve $z_{1}$ and $z_{N} . K_{N}(0,0) \equiv Z$ provides the normalization. Appropriate average reads

$$
\begin{aligned}
\left.\left\langle z_{k}^{2}\right\rangle\right|_{z_{1}=z_{N}=0} & =\int d \bar{z}_{1} d x_{2} d y_{2} \ldots d y_{N-1} d \bar{z}_{N}\left(x_{k}+i y_{k}\right)^{2} \exp \left(-X^{T} R X\right) / Z \\
& =\frac{1}{2}\left(R_{2 k-2,2 k-2}^{-1}+i\left(R_{2 k-2,2 k-1}^{-1}+R_{2 k-1,2 k-2}^{-1}\right)-R_{2 k-1,2 k-1}^{-1}\right),
\end{aligned}
$$

and can be easily calculated. After the first limit (5.6) it simplifies to

$$
\lim _{1}\left\langle z_{k}^{2}\right\rangle=\frac{i}{2 d} \frac{(k-1)(N-k)}{N-1} \stackrel{N \rightarrow \infty}{\longrightarrow} \frac{i \hbar}{m} \frac{t(T-t)}{T},
$$

which is just the discretized version of (6.3), since

$$
(N-1) \epsilon=T, \quad(k-1) \epsilon=t .
$$

Again the weight is not entirely positive because of the integration over two complex (but $2 N-4$ real) variables. As said above this is the consequence of the zero mode and how it was fixed. It remains to be seen if other ways of dealing with translational symmetry could change that.

The next applications is free of this problem.

\subsection{A harmonic oscillator}

There is no zero mode here, therefore we define now the average over all periodic trajectories,

$$
\left\langle x^{2}(T)\right\rangle=\left\langle x^{2}(0)\right\rangle=\frac{\int d x x^{2} K(x, x ; T)}{\int d x K(x, x ; T)},
$$

which measures the width of a periodic Minkowski trajectory with the length $T$. This is the different observable than was considered in the free particle case. With

$$
K\left(x_{b}, x_{a} ; T\right) \sim \exp \left\{\frac{i}{\hbar} \frac{m \omega}{2 \sin \omega T}\left(\left(x_{a}^{2}+x_{b}^{2}\right) \cos \omega T-2 x_{a} x_{b}\right)\right\},
$$

one easily obtains

$$
\left\langle x^{2}(T)\right\rangle=-\frac{i \hbar T}{4 m} \frac{\cot \frac{\omega T}{2}}{\frac{\omega T}{2}} .
$$

In our framework, and upon the discretization, this is given by the straightforward average over the positive distribution (4.5) of $2 N$ real variables $X^{T}=\left(x_{1}, y_{1}, \ldots, x_{N}, y_{N}\right)$

$$
\left\langle z_{1}^{2}\right\rangle=\frac{1}{Z} \int \prod_{j=1}^{N} d x_{j} d y_{j}\left(x_{1}+i y_{1}\right)^{2} \exp \left\{-X^{T} M X\right\} .
$$




\begin{tabular}{|cc|}
\hline$N$ & $P_{N}(x) / Q_{N}(x)$ \\
\hline 5 & $\frac{\left(x^{2}-2 x-4\right)\left(x^{2}+2 x-4\right)}{x^{2}\left(x^{4}-20 x+80\right)}$ \\
8 & $\frac{7\left(128 x^{8}-12544 x^{6}+384160 x^{4}-3764768 x^{2}+5764801\right)}{32 x^{2}\left(x^{2}-49\right)\left(2 x^{2}-49\right)\left(8 x^{4}-392 x^{2}+2401\right)}$ \\
11 & $\frac{5\left(x^{5}-5 x^{4}-100 x^{3}+375 x^{2}+1875 x-3125\right)\left(x^{5}+5 x^{4}-100 x^{3}-375 x^{2}+1875 x+3125\right)}{2 x^{2}\left(x^{10}-275 x^{8}+27500 x^{6}-1203125 x^{4}+21484375 x^{2}-107421875\right)}$ \\
\hline
\end{tabular}

Table 2. Dispersion of a Minkowski trajectory calculated from the positive representation (6.6) for few discretizations.

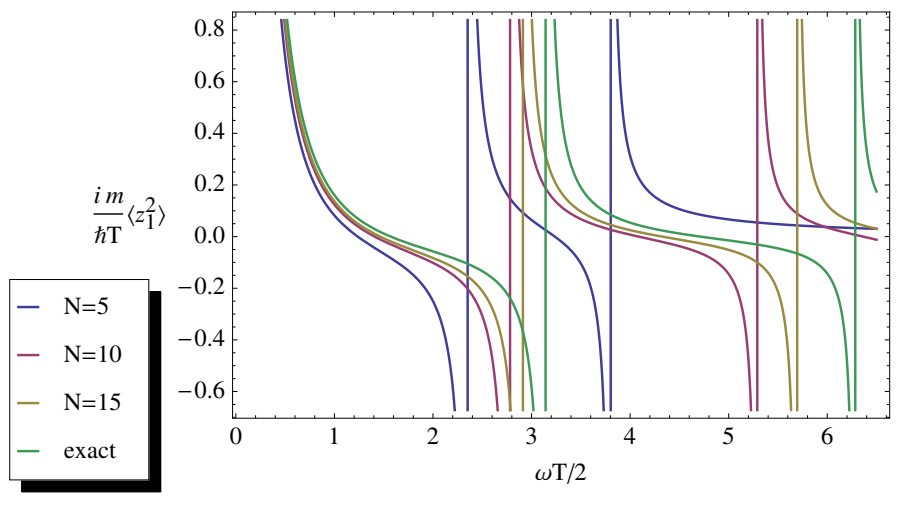

Figure 1. Convergence of (6.7) to (6.5).

Gaussian average is again given by the same combination of matrix elements as in (6.4) but with the original matrix $M$. In particular $\left\langle z_{k}^{2}\right\rangle$ is independent of $k$ due to the invariance under time shifts.

The explicit expression for (6.6) in terms of $a, b$ and oscillator parameters is somewhat messy. However upon taking the first limit along the trajectory (5.8) it simplifies to

$$
\lim _{\nu \rightarrow 0}\left\langle z_{1}^{2}\right\rangle=-\frac{i \hbar T}{m} \frac{P_{N}(\omega T / 2)}{Q_{N}(\omega T / 2)} .
$$

The first few polynomials $P_{N}(x)$ and $Q_{N}(x), x=\omega T / 2$, are listed in table 2. They gradually build up $\cot (x) / 4 x$ with increasing $N$, cf. figure 1 , and one readily recovers the continuum result (6.5) at $N \rightarrow \infty$.

The transition from (6.7) to (6.5) is of course well known since the classic works by Feynman $[20,21]$. The novel element here is that (6.7) was obtained as the probabilistic average of a suitable (i.e. complex) observable over the positive distribution (4.6).

On the other hand, at finite $N$, the action (4.5) has one negative mode as discussed in section 3. Nevertheless the inverse matrix exists meaning that the divergent integral over the negative mode is defined by the analytic continuation. This analytic continuation provides a regularization of the divergence and leads finally to the correct result. Moreover, by applying the original trick [14] for a second time, and to the negative mode only, one could construct the positive and normalizable distribution which would allow for statistical calculation of the above and other averages. 


\subsection{Charged particle in a constant magnetic field}

This is again the textbook problem in elementary path integrals. It is also the simplest example where Wick rotation does not render the positive Boltzmann factor. Since the action is again quadratic, it should be possible to construct the corresponding positive density $P_{N}(x, y)$ similarly to the previous examples. Here we shall follow the simpler approach. It is known since the time of Landau that the problem can be reduced to that of a shifted harmonic oscillator. To use this observation we need to establish the Landau reduction on the level of Feynman propagators. Begin with the phase space path integral

$$
K^{B}\left(\vec{x}_{b}, \vec{x}_{a}, T\right)=\int \mathcal{D} \vec{p}(t) \mathcal{D} \vec{x}(t) \exp \left\{\frac{i}{\hbar}(\vec{p} \cdot \dot{\vec{x}}-H(\vec{p}, \vec{x}))\right\}
$$

In the gauge used by Landau $(\vec{A}=B(0, x, 0))$ the Hamiltonian reads

$$
H=\frac{1}{2 m} p_{x}^{2}+\frac{1}{2 m}\left(p_{y}-\frac{e B}{c} x\right)^{2}
$$

and one readily obtains from $(6.8), O=c p_{y} / B e$,

$$
K^{B}\left(\vec{x}_{b}, \vec{x}_{a} ; T\right)=\int d O \exp \left\{\frac{i}{\hbar} m \omega O\left(y_{b}-y_{a}\right)\right\} K_{O}^{\mathrm{HO}}\left(x_{b}, x_{a} ; T\right),
$$

where $K_{O}^{\mathrm{HO}}\left(x_{b}, x_{a} ; T\right)$ is the kernel for the one dimensional (in $\left.x\right)$ harmonic oscillator located at $x_{0}=O$. The integral is again gaussian and is saturated by the classical position of the center of oscillations

$$
O_{x}=\frac{1}{2}\left(x_{a}+x_{b}\right)+\frac{1}{2} \cot \frac{\omega T}{2}\left(y_{b}-y_{a}\right) .
$$

Consequently the propagator reads

$$
K^{B}\left(\vec{x}_{b}, \vec{x}_{a} ; T\right) \sim \exp \left\{\frac{i}{\hbar} m \omega O_{x}\left(y_{b}-y_{a}\right)\right\} K_{O_{x}}^{\mathrm{HO}}\left(x_{b}, x_{a} ; T\right) .
$$

This (a) corresponds exactly to the Landau solution of the Schrödinger equation by separation of variables and (b) after a simple algebra reproduces the Feynman result in the gauge employed by Landau

$$
K_{\mathrm{LG}} \sim \exp \left\{\frac{i m}{2 \hbar}\left(\frac{\omega}{2} \cot \frac{\omega T}{2}\left(\left(x_{b}-x_{a}\right)^{2}+\left(y_{b}-y_{a}\right)^{2}\right)+\omega\left(x_{a}+x_{b}\right)\left(y_{b}-y_{a}\right)\right)\right\} .
$$

Now the reduction (6.9) can be used to extend our positive representation (4.6) also to the case of an external magnetic field. Take as an example the average position of a quantum particle at time $0<t<T$ assuming that at $t=0$ and $t=T$ it was at $\vec{x}_{a}$ and $\vec{x}_{b}$ respectively

$$
\langle\vec{x}\rangle_{B}=\int d^{2} x K\left(\vec{x}_{b}, \vec{x} ; T-t\right) \vec{x} K\left(\vec{x}, \vec{x}_{a} ; t\right) / K\left(\vec{x}_{b}, \vec{x}_{a} ; T\right)=x_{x_{a}, x_{b}, T}^{c l}(t) .
$$


Since the problem is gaussian the well known, gauge invariant, answer is just the classical trajectory which satisfies above conditions. To see how our representation works in this case one can use (6.9) to rewrite (6.10) as harmonic oscillator averages

$$
\begin{aligned}
\langle x(t)\rangle_{B} & =\langle x(t)\rangle_{O=O_{x}}, \\
\langle y(t)\rangle_{B} & =\langle y(t)\rangle_{O=O_{y}} .
\end{aligned}
$$

The second line is derived in yet another gauge where the magnetic field problem reduces to the oscillator along the $y$ direction with the analogous classical expression for the center of $y$ oscillations.

To complete the construction we only need to extend the positive density (4.6) such that it describes a shifted harmonic oscillator. This is done by simply adding linear terms to the action

$$
S_{N}(z, \bar{z}) \rightarrow S_{N}(z, \bar{z})+\sum_{i} e^{*} z_{i}+e \bar{z}_{i}
$$

or by just shifting $z \rightarrow z_{i}-z_{c}$ and $\bar{z} \rightarrow \bar{z}_{i}-z_{c}^{*}$. The new density $P_{N}$ remains positive and normalizable as before.

Calculation of the appropriate averages in the new representation is now a simple exercise and proceeds analogously to previous applications, e.g. (6.3). To avoid a confusion with the primordial cartesian coordinates $x$ and $y$ in (6.11), we have renamed the real and imaginary parts of their complex extensions $z_{k}$, i.e. $z_{k}=u_{k}+i v_{k}, \bar{z}_{k}=u_{k}-i v_{k}$. Since the end-points are again fixed the averages are taken over $2 N-4$ "positive" variables $u_{i}, v_{i}$ and two complex $\bar{z}_{1}$ and $\bar{z}_{N}$. Compared to (6.1) there is an additional source term in the action caused by the shift (6.12). The final result obtains after taking the scaling limit $\left(\lim _{1}\right)$ defined in (5.8) followed by the usual continuum limit.

$$
\langle x(t)\rangle=\lim _{N \rightarrow \infty} \lim _{\nu \rightarrow 0}\left\langle z_{k}\right\rangle=\lim _{N \rightarrow \infty} \lim _{\nu \rightarrow 0}\left\langle u_{k}+i v_{k}\right\rangle_{P_{N}\left(\bar{z}_{1}, u^{\prime} s, v^{\prime} s, \bar{z}_{N}\right)} .
$$

In figure 2 a sample of averages, after taking the first limit, is shown and compared with the two corresponding classical trajectories, which differ by the choice of the $\omega T$. Convergence with $N$ is satisfactory and not surprising. The main point, however, is that the averages are calculated over the new, positive in the fully inclusive case, distribution and they converge in the first limit to the standard Feynman discretization.

\section{Summary and conclusions}

Problems with complex solutions of the Langevin equations can be avoided by the direct construction of pairs of corresponding complex and positive densities, without any reference to complex stochastic processes or Fokker-Planck equations. This is done in sections 3 and 4 for the gaussian model and for its simple nonlinear modification. ${ }^{3}$ As a byproduct the well known solution of the gaussian model was generalized, thereby providing a positive representation for an arbitrary complex dispersion parameter. In particular it works also for the purely imaginary slope.

\footnotetext{
${ }^{3}$ See $[14]$ for some details.
} 


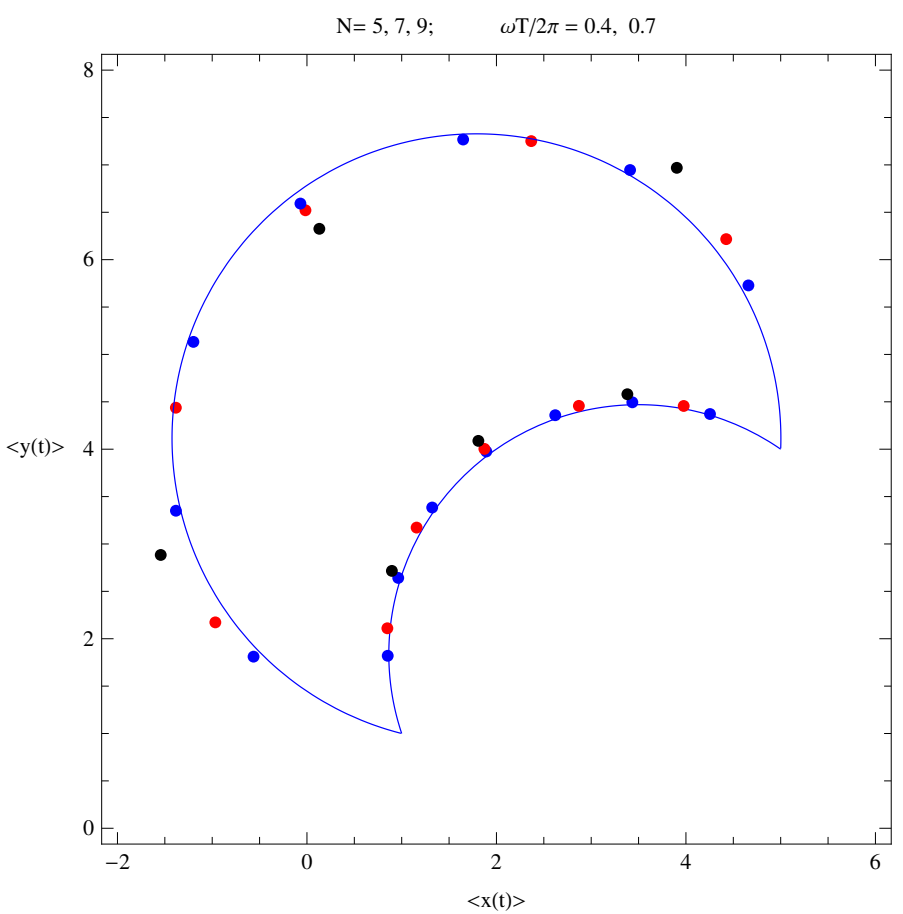

Figure 2. Two classical trajectories of a charged particle in a constant magnetic field (solid lines). Points represent the first limit of averages (6.11) calculated with shifted positive density (4.6), (6.12) for finer and finer discretizations.

In is also noteworthy that the method works successfully in a nonlinear case as shown in section 4. Hence it is more general than could have been inferred from the gaussian applications only.

In the sequel the gaussian solution is generalized to many variables and used to construct the positive representation for gaussian path integrals directly in the Minkowski time. For the infinite number of degrees of freedom existence of the continuum limit is not trivial and is discussed in some details. In particular the couplings appearing in the new representation have to be tuned in a well defined way to assure the existence of the continuum limit.

The procedure is then successfully applied to the three textbook quantum mechanical problems: a free particle, a harmonic oscillator and a particle in a constant, external magnetic field. The latter is the simplest prototype of a Wilson loop and is known for its lack of a positive weight after the Wick rotation. Consequently the present construction provides the first positive representation for this important physical problem.

Many questions remain open, even in the context of above simple cases. For example, how fast is the first limit achieved in practice, how this depends on $N$, is there a more optimal way to combine the first limit with the continuum limit, etc.

Obviously one would like to generalize the present scheme to non-gaussian systems. The nonlinear example solved in section 4 shows, that the new structure is not necessarily restricted to the gaussian case. A simple generalizations of this model to higher degrees of 
nonlinearity can be constructed. An example is given by the action being a higher than two (but even) power of the exponent of (2.1). At the same time we point out that the general proofs of the positive representations exist in the literature [16, 18]. Hence the above example may be thought of as providing the concrete realization of these general principles.

Related with this is a mathematical problem to what extent can the sum rule (1.4), together with positivity and normalizability conditions, determine $P$ from a complex weight $\rho$. A possible strategy to attack this question might be based on the continuity and/or deformation principle: for gaussian case the corresponding densities are known explicitly. A small deformation of the complex density should result in a small change of the corresponding $P$. It is conceivable that repeating this procedure many times would lead to the final $\mathrm{P}$ representing the more general, also non-gaussian $\rho$.

Certainly all above questions should be studied more systematically and separately. At the same time the very fact that the present formulation provided for the first time the positive representation of Minkowski path integrals for some simple, yet concrete physical systems ${ }^{4}$ is nontrivial and interesting.

Barring above, a host of further problems and applications suggests itself: generalization to compact integrals, nonlinear and nonabelian couplings, fermionic integrals, as well as extensions to the field theory, are only few examples. We are looking forward to study some of them.

Finally, an intriguing analogy may be enjoyed. Basically the positivity is achieved by duplicating the number of variables. In these variables, Minkowski weights become positive as long as boundary conditions for Feynman paths are not specified, i.e. when only traces of evolution operators (and/or their moments) are required. Moreover, path integrals in above variables involve a new limiting transition, which may lead, via the saddle point mechanism, to the dominance of a concrete class of trajectories. All this resembles to some extent the celebrated history of hidden variables. At the same time we strongly emphasize that none of the sacred principles of quantum mechanics is violated. The standard, complex quantum amplitudes emerge upon suitable integrations over half of above variables with the usual fixed boundary conditions. Therefore the quantum interference is not violated in any way. Similarly, even though some couplings between new variables indeed have to tend to infinity in the first limit, there are others which remain constant and are in fact $O(1 / \hbar)$, hence they drive the usual quantum fluctuations of a system.

Interestingly ref. [16] concludes with similar considerations, which however are more hypothetical due to the lack of the continuum limit analysis. It will be very interesting to study how the existence of the latter restricts some scenarios mentioned there.

It remains to be seen if the new structure of quantum amplitudes exposed in this article turns out to be of general interest only, if it is more fundamental (in the sense of the closing speculations of ref. [16]), or last but certainly not least, if it can be generalized to more complicated systems.

\footnotetext{
${ }^{4}$ Notably including a motion of a particle in an external magnetic field - the problem which did not have positive representation even after the Wick rotation.
} 


\section{Acknowledgments}

Stochastic interpretation of Feynman path integrals has been studied on more general grounds in refs. [22, 23].

I would like to thank Owe Philipsen and Erhard Seiler for the discussion.

Open Access. This article is distributed under the terms of the Creative Commons Attribution License (CC-BY 4.0), which permits any use, distribution and reproduction in any medium, provided the original author(s) and source are credited.

\section{References}

[1] G. Parisi and Y. Wu, Perturbation theory without gauge fixing, Sci. Sin. 24 (1981) 483 [INSPIRE].

[2] P.H. Damgaard and H. Huffel, Stochastic Quantization, Phys. Rept. 152 (1987) 227 [INSPIRE].

[3] G. Parisi, On complex probabilities, Phys. Lett. B 131 (1983) 393 [InSPIRE].

[4] J.R. Klauder, Coherent State Langevin Equations for Canonical Quantum Systems With Applications to the Quantized Hall Effect, Phys. Rev. A 29 (1984) 2036 [INSPIRE].

[5] E. Seiler, D. Sexty and I.-O. Stamatescu, Gauge cooling in complex Langevin for QCD with heavy quarks, Phys. Lett. B 723 (2013) 213 [arXiv:1211.3709] [INSPIRE].

[6] G. Aarts, F. Attanasio, B. Jäger, E. Seiler, D. Sexty and I.-O. Stamatescu, The phase diagram of heavy dense QCD with complex Langevin simulations, Acta Phys. Polon. Supp. 8 (2015) 405 [arXiv : 1506. 02547] [INSPIRE].

[7] J. Ambjørn and S.K. Yang, Numerical Problems in Applying the Langevin Equation to Complex Effective Actions, Phys. Lett. B 165 (1985) 140 [INSPIRE].

[8] J. Ambjørn, M. Flensburg and C. Peterson, The Complex Langevin Equation and Monte Carlo Simulations of Actions With Static Charges, Nucl. Phys. B 275 (1986) 375 [InSPIRE].

[9] R.W. Haymaker and J. Wosiek, Complex Langevin simulations of non-Abelian integrals, Phys. Rev. D 37 (1988) 969 [INSPIRE].

[10] J. Glesaaen, M. Neuman and O. Philipsen, Heavy dense QCD from a 3d effective lattice theory, arXiv:1511.00967 [INSPIRE].

[11] J. Bloch, J. Mahr and S. Schmalzbauer, Complex Langevin in low-dimensional QCD: the good and the not-so-good, arXiv: 1508.05252 [INSPIRE].

[12] G. Aarts, E. Seiler and I.-O. Stamatescu, The Complex Langevin method: When can it be trusted?, Phys. Rev. D 81 (2010) 054508 [arXiv:0912.3360] [InSPIRE].

[13] G. Aarts, F.A. James, E. Seiler and I.-O. Stamatescu, Complex Langevin: Etiology and Diagnostics of its Main Problem, Eur. Phys. J. C 71 (2011) 1756 [arXiv:1101.3270] [INSPIRE].

[14] J. Wosiek, Beyond complex Langevin equations I: two simple examples, arXiv:1511.09083 [INSPIRE].

[15] H. Okamoto, K. Okano, L. Schulke and S. Tanaka, The Role of a Kernel in Complex Langevin Systems, Nucl. Phys. B 324 (1989) 684 [INSPIRE]. 
[16] D. Weingarten, Complex probabilities on $R^{N}$ as real probabilities on $C^{N}$ and an application to path integrals, Phys. Rev. Lett. 89 (2002) 240201 [quant-ph/0210195] [INSPIRE].

[17] L.L. Salcedo, Representation of complex probabilities, J. Math. Phys. 38 (1997) 1710 [hep-lat/9607044] [INSPIRE].

[18] L.L. Salcedo, Existence of positive representations for complex weights, J. Phys. A 40 (2007) 9399 [arXiv:0706.4359] [INSPIRE].

[19] G. Aarts, F.A. James, J.M. Pawlowski, E. Seiler, D. Sexty and I.-O. Stamatescu, Stability of complex Langevin dynamics in effective models, JHEP 03 (2013) 073 [arXiv:1212.5231] [INSPIRE].

[20] R.P. Feynman, Space-time approach to nonrelativistic quantum mechanics, Rev. Mod. Phys. 20 (1948) 367 [INSPIRE].

[21] R.P. Feynman and A.R. Hibbs, Quantum Mechanics and Path Integrals, McGraw-Hill Inc., New York (1965).

[22] Z. Haba, Stochastic interpretation of Feynman path integral, J. Math. Phys. 35 (1994) 6344 [INSPIRE].

[23] Z. Haba, Semiclassical stochastic representation of the Feynman integral, J. Phys. A 27 (1994) 6457. 